\title{
The Digital Image Restoration Method Based on a comprehensive evaluation of subjective and objective
}

\author{
YaojiaHuang ${ }^{1}$,TaoCheng ${ }^{2}$,PingFeng ${ }^{3}$,XiaoboPeng ${ }^{4}$ \\ ${ }^{1}$ Apartment 20, Guimiao Village, Shenzhen University, Shenzhen, China \\ ${ }^{2}$ Mechatronics and Control Engineering, Shenzhen University, Shenzhen, \\ China \\ ${ }^{3}$ Mechatronics and Control Engineering, Shenzhen University, Shenzhen, \\ China \\ ${ }^{4}$ Mechatronics and Control Engineering, Shenzhen University, Shenzhen, \\ China
}

\begin{abstract}
Given the importance of image quality evaluation in the field of image acquisition and processing, and for the most commonly used method for objective evaluation and subjective evaluation method in the limitations of practical application, this paper presents a comprehensive image quality evaluation method based on objective and subjective. To address the digital image restoration repair effect of uncertainty exists in low efficiency. The simulation results show that the digital image evaluation, repair method proposed in this paper could significantly improve the efficiency and quality of image restoration. A comparative study of the text in a variety of digital image evaluation and repair methods, as well as the related simulation.
\end{abstract}

Keyword: Image quality; based on objective and subjective image quality comprehensive evaluation; digital image restoration; Simulation

\section{Introduction:}

All image processing needs an appropriate evaluation criteria to assess the image quality, Which is usually divided into subjective and objective methods. Subjective evaluation refers to the observer on their own sense of the results directly to repair the image quality evaluation method. Subjective evaluation is one of the most popular of the most important image quality assessment method in image repair. Subjective evaluation of the advantages is to facilitate the implementation and compliance with human visual characteristics, the disadvantage is affected by the observer background knowledge, observation and environmental factors, the results poor stability, portability is also poor, time-consuming, difficult to meet the real-time image quality assessment requirements. Objective evaluation to assess digital avoid the visual impact of 
interference in a digital image of objective criteria now in many of these assessment methods. Such as Mean Square Error (MSE) and Peak Signal to Noise Ratio (PSNR) and other methods. As the flexibility of no reference image quality assessment method, which is gaining more and more attention ,among several objective image quality assessment method.

Image restoration is to the image information of the defect area filled with process information, and can not make the observer perceive images that have lost or has been repaired. Currently there are two class image restoration techniques: one is used to repair small-scale digital image defect repair technique. This technique uses the area to be repaired edge information while using a coarse-to-fine approach to the estimation of the direction of the illumination line, and uses the dissemination of information dissemination mechanism to be repaired within the region, in order to get a better fix results . The method of this class can be referred to as the variational inpainting algorithm based on the PDE. Another one is used to fill in the image chunks missing image full complement of information technology. With block-based texture synthesis technique to fill the missing information, the main idea of this kind of algorithm is to first select a pixel from the border area to be repaired, but to this point as the center, according to the texture of the image, select the appropriate size the texture block, then in the area around is seeking to be the most similar texture patches.

\section{The basic idea of the primary process}

\subsection{The basic idea}

Compared analysis of no reference evaluation with reference evaluation in Objective evaluation of image, to explore the establishment of a no-reference evaluation based on the largest possible scope of image evaluation system. In order to evaluate the effect of several different image restoration algorithm, to choose better and more efficient image restoration.

\subsection{The main process}

First, a comparison of several different objective evaluation criteria, and then each subjected to the relevant algorithm simulation. Secondly, the image restoration algorithm were compared, and the corresponding image restoration algorithm simulation. Finally, combined with image evaluation and image restoration algorithms comprehensive comparative analysis, image evaluation system to evaluate the effect of image restoration, image repair to verify the correctness and applicability of image evaluation system.

\section{Objective evaluation of the quality standard image restoration}

Objective quality assessment refers to the use of one or more images of metrics, the mathematical model associated with the image quality so that the computer automatically calculated image quality, the goal is the objective and subjective evaluation results are consistent feel. Whether the extent of the 
original image reference and reference objective quality assessment can be divided into the following three types: 1) the full reference method (Full Reference, FR), requires a full evaluation of the original image as a reference; 2) semi-Reference methods (Reduced Reference, RR), part of the information required of the original image as an evaluation of reference; 3) no reference method (No Reference, NR), without the aid of any reference images, the images themselves rely variety of information to be evaluated for quality evaluation. Because the full reference method and the reference method requires half the original image information as a reference, and the results obtained by these two methods are often not well reflect the subjective feelings, so no reference method is being more and more attention.

2.1 Full or Reduced-reference evaluation methods

The simplest method for evaluating the full reference Mean Square Error (MSE) and Peak Signal to Noise Ratio (PSNR), which direct the pixel gray values of the reference image and the distorted image are calculated, simple calculation, meaning clear, but not well with people's subjective feelings remain consistent. Suppose two corresponding image size $M^{*} N$, respectively, image rows and columns, where $f(i, j)$ and $\tilde{f}(i, j)$ are the original image and the fixed image. Here are concentrated image restoration evaluation criteria:

1) Definition of MSE

$$
\mathrm{MSE}=\frac{1}{\mathrm{MN}} \sum_{i=1}^{M} \sum_{j=1}^{N}[\mathrm{f}(\mathrm{i}, \mathrm{j})-\tilde{\mathrm{f}}(\mathrm{i}, \mathrm{j})]^{2}
$$

For the mean square error for a color image, and the gray image is slightly different, each R, G, B channels are respectively calculated variance, the variance value shall give three first divided by the sum obtained by adding $M^{*} N$, and then divided by 3 , the result is the average standard deviation of the color image. This value is smaller, the better the image restoration.

2) Definition of PSNR

$$
\text { PSNR }=10 \log \left\{\frac{255^{2}}{\frac{1}{\mathrm{MN}} \sum_{i=1}^{M} \sum_{j=1}^{N}[\mathrm{f}(\mathrm{i}, \mathrm{j})-\tilde{\mathrm{f}}(\mathrm{i}, \mathrm{j})]^{2}}\right\}
$$

PSNR is repaired after the initial image and the image of the mean squared error measure with respect to the square of the maximum pixel value is expressed in decibels $\mathrm{dB}$. PSNR bigger the better.

2.2 No-reference evaluation methods

Currently, no reference image distortion metric is generally for one or a few types of distortion, the image of a type of distortion metrics and subjective test value by combining results of objective quality assessment values. NR-NIQE (Naturalness Image Quality Evaluator) simulation algorithm uses a no-reference image quality assessment based on machine learning methods.

Machine learning method based on the basic idea of no-reference image quality assessment algorithm is: in the training phase, the subjective test values are classified according to the quality of the image; extracting feature vectors reaction image quality, this feature vector and its corresponding image quality The build quality classification category. During the testing phase, will be drawn in the same way as input feature vectors classifier, the output value of the 
classifier is the objective evaluation of the value of the image quality. Features of these methods can be achieved by means of machine learning research, quality improvement and objective evaluation results.

\section{Techniques of digital image restoration based Variational PDE}

Variational PDE is an image restoration technology research and application of early repair the main method, the basic principle is the use of mathematical diffusion equation repair information to be spread around the area to be repaired in order to achieve the damaged area of the image restoration. Among them, the overall total variation (TV) model variational algorithm is a branch belonging to rate $\mathrm{PDE}$ algorithms. While the algorithm can effectively maintain the edge, but also to remove noise interference, is different to the diffusion algorithm.

3.1 Algorithm principle

TV model is the use of the neighborhood area to be repaired to repair the defect image pixel information, and strive to make the image as much as possible after the repair achieve realistic results. The main idea is to find an energy function is minimized by the extreme value of the energy function to achieve the restoration of damaged images. Formula (3.1) as follows:

$$
\min E_{\mathrm{tv}}\left[u \mid \mathrm{u}^{0}, D\right]=\int_{\Omega}|\nabla u| \mathrm{dx}+\frac{\lambda}{2} \int_{\Omega \backslash \mathrm{D}}\left(u-u^{0}\right)^{2} \mathrm{dx}
$$

Where, $\Omega$ for the entire image area, $\mathrm{D}$ is the area to be repaired.

According to the variational principle, the corresponding Euler-Lagrange equation (3.2):

$$
\begin{aligned}
& \operatorname{div}[\nabla \mathrm{u}|\nabla \mathrm{u}|]-\lambda_{D}(x)\left(u-u^{0}\right)=0 \\
& \lambda_{D}(x)=\lambda \cdot I_{\Omega \backslash \mathrm{D}}(x)=\left\{\begin{array}{lr}
\lambda & (\forall x \in \Omega \backslash \mathrm{D}) \\
0 & (\forall x \in \mathrm{D})
\end{array}\right.
\end{aligned}
$$

Formula (3.2) is also equivalent to the formula (3.4).

$$
\frac{\partial u}{\partial t}=\nabla \cdot\left[\frac{\nabla u}{|\nabla u|}\right]+\lambda_{D}(x)\left(u-u^{0}\right)
$$

This is a nonlinear diffusion equation, with the change of time $t$ when $\partial \mathrm{I} / \partial \mathrm{t}$ tends to zero, you can get the minimum value of $\mathrm{u}$. Boundary $\partial \Omega$ biased conditions must be met: $\partial \mathrm{u} / \partial \overrightarrow{\mathrm{v}}$, where $\overrightarrow{\mathrm{v}}$ for normal vector at the boundary.

Due to the smooth region $|\nabla u|$ will be very small, there may even tend to zero, it is generally used $\nabla \cdot[\nabla \mathrm{u} /|\nabla \mathrm{u}|]$ alternative $\nabla \cdot\left[\nabla \mathrm{u} /|\nabla \mathrm{u}|_{\varepsilon}\right]$ to avoid the denominator is zero, which $|\nabla u|_{\varepsilon}=\sqrt{\varepsilon^{2}+|\nabla u|^{2}}, \varepsilon$ is a small positive parameter.

This formula (3.1) becomes:

$$
\min E_{\mathrm{tv}}\left[u \mid \mathrm{u}^{0}, D\right]=\int_{\Omega}|\nabla \mathrm{u}|_{\varepsilon} \mathrm{dx}+\frac{\lambda}{2} \int_{\Omega \backslash \mathrm{D}}\left(u-u^{0}\right)^{2} \mathrm{dx}
$$

So as to treat the repair area pixel interpolation, continuous loop until repair is complete. 


\section{Techniques of digital image restoration based Sample texture synthesis}

"sample Texture synthesis" are given a new map based on synthetic texture images, so the texture image and texture samples of newly synthesized have roughly the same visual effect. Substantive defect image repair the defect area is the use of image information to fill in the gaps around the defect information, so that the two have the same visual effect. Which, Criminisi algorithm repair strategy is to follow the above-mentioned one of the most representative sample image restoration algorithm based on texture synthesis.

4.1 Algorithm principle

Criminisi algorithm damaged image before the repair process requires the user to determine the image aim area to be repaired, the general use of a particular color to the damaged area marked as shown.

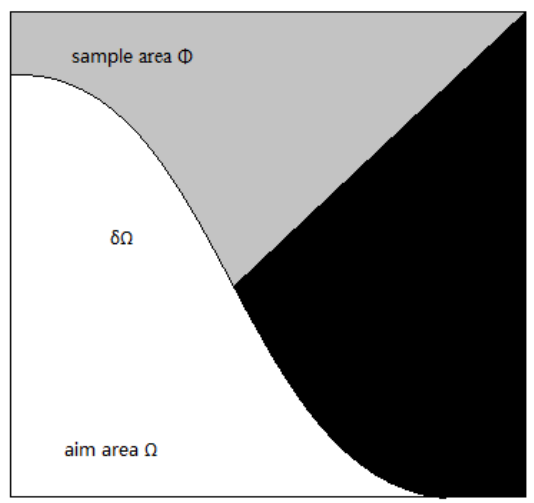

Fig 4-1 Criminisi algorithm schematics

Wherein, the entire original image is represented by I damaged, the damaged area of the damaged original image needs to be repaired, the damaged area of the boundary, for the original image in the damaged region of the sample intact, $\mathrm{p}$ is damaged at a boundary region having the maximum repair priority point, the image point represented by the blocks of q samples of known area and the point $\mathrm{p}$ where the repair block to be most similar block (i.e., the best matching block of samples). The algorithm steps are as follows:

First, calculate the priority.

The priority of point $\mathrm{p}$ is $\mathrm{P}(\mathrm{p})=\mathrm{C}(p) \mathrm{D}(p)$, wherein, $\mathrm{C}(p)$ represents a block of confidence, $\mathrm{D}(p)$ is called a data item, $\mathrm{C}(p)$ and $\mathrm{D}(p)$, respectively defined by the formula:

$$
\begin{aligned}
\mathrm{C}(p) & =\frac{\sum_{q \in \Psi_{p} \cap(I-\Omega)} c(\mathrm{q})}{\Psi_{p}} \\
\mathrm{D}(p) & =\frac{\left|\nabla I_{\mathrm{p}} \perp n_{p}\right|}{\alpha}
\end{aligned}
$$

$\left|\Psi_{p}\right|$ is $\Psi_{p}$ area, which is within the template of the total number of pixels. $\alpha$ is a normalization factor. $n_{p}$ is orthogonal to the boundary at point $\mathrm{p} \delta \Omega$ 
unit orthogonal vectors. $\perp$ pointed out positive AC operation. $c(\mathrm{q})$ for pixel $\mathrm{q}$ confidence value. Those must meet the initialization condition:

$$
c(\mathrm{q})=\left\{\begin{array}{lr}
1 & q \in \Phi \\
0 & q \in \mathrm{I}-\Phi
\end{array}\right.
$$

Formula (4.1) and (4.3) show that, p pixels located block to be repaired, contain the more known information of the pixel, then the block of the higher degree of confidence, on the contrary, the less pixel including known information, the lower the confidence level of this block. (4.2) where, $\alpha$ is "normalization factor", usually $\alpha$ value 255 . $\nabla \mathrm{I}_{\mathrm{p}} \perp$ indicates a direction perpendicular to the direction of the gradient with point p. $\mathrm{n}_{\mathrm{p}}$ for defect area boundary $\delta \Omega$ the normal vectors of point $\mathrm{p} . \nabla \mathrm{I}_{\mathrm{p}} \perp$ line direction of illumination point $\mathrm{p}$. It reflects the structure of the extended trend of the image. this feature is applied to the image to repair, to ensure that the structure of the defect area filled. As can be seen from this formula, the greater the intensity of illumination line point $\mathrm{p}$, the angle of illumination of the line and the normal vector is smaller, the data items $D(p)$ the value of the greater, then the priority value of the point $p$, the more large, repair the defect blocks to be p points based on priority to get filled.

Second, the search for the best matching block and fill.

By calculating the priority is determined to be repaired after block, Criminisi algorithm on the use of global search method known sample area (no-damaged region) Search block to be repaired and the highest similarity blocks, in other word, the best matching block samples. Hypothesis "has the highest priority of the center pixel template" for $\mathrm{p}$, the corresponding block is the template block $\Psi_{p}$ be repaired, to search for the best in the area matching block $\Psi_{\hat{q}}$ not damaged, just compare $\mathrm{d}\left(\Psi_{p}, \Psi_{\hat{q}}\right)$, make $\mathrm{d}\left(\Psi_{p}, \Psi_{\hat{q}}\right)$ is the smallest of the best matching block matching block $\Psi_{\hat{q}}$. Here $\mathrm{d}\left(\Psi_{p}, \Psi_{\hat{q}}\right)$ represents $\Psi_{p}$ and $\Psi_{\hat{q}}$ within two blocks of pixels corresponding to the square of the difference between the pixel color values and, commonly called "Euclidean distance.". After Finding the corresponding point $\hat{q}$, replace $\Psi_{\hat{q}}$ with $\Psi_{p}$ pixel values corresponding to complete the process of filling the block to be repaired.

Third, update the confidence values.

After filling the block $\Psi_{p}$ to be repaired, $\Psi_{p}$ becomes repaired good block. A new round of the repair process will be carried out not defect area (the original area to be repaired accordingly reduced), this time to update this block confidence within the dots, and update the formula such as (4.4). It is assumed $\Psi_{p}$ for "just reconditioned block".

$$
\mathrm{c}(\dot{\mathrm{p}})=\mathrm{c}(\mathrm{p}), \forall \mathrm{p} \in \Psi_{p} \cap \Omega
$$

After more than three steps, even completed a repair process. And so forth, until the filling to repair a damaged area complete after the end of the execution.

\section{The simulation results and analysis}

According to the algorithm using Matlab prepared a comparative experiment image restoration. 


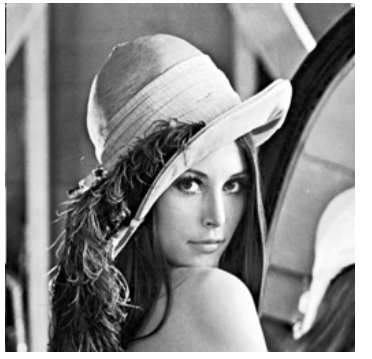

(a) original image1

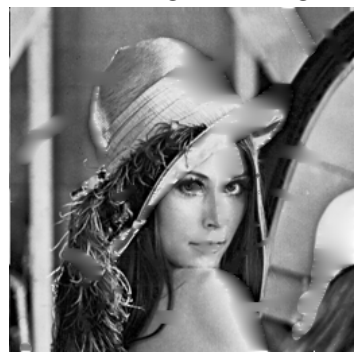

(c) TV Repair effect of image1

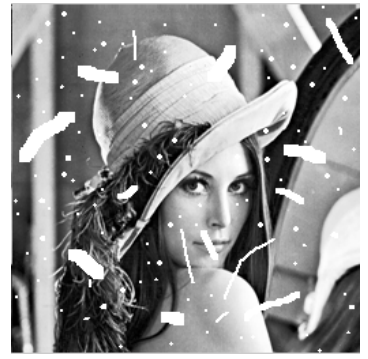

(b) damage image 1

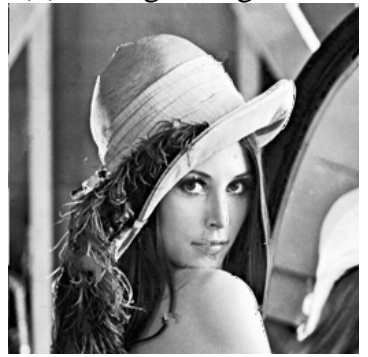

(d) Criminisi Repair effect of image1

Fig 5-1 Repair effect of contrast of distorted picture1 (FR)

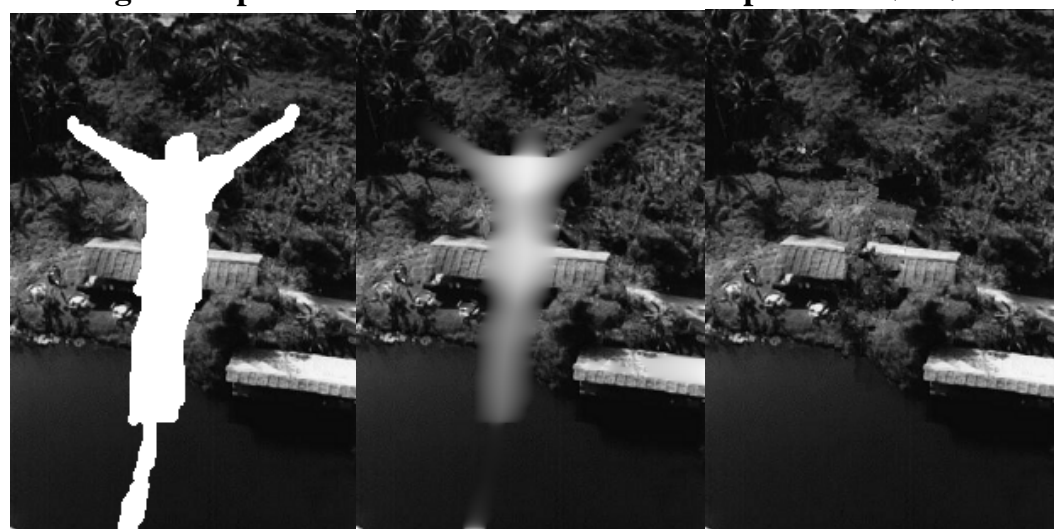

(a) original image2

(b) TV Repair effect of image2 (c) Criminisi Repair effect of image 2

Fig 5-2 Repair effect of contrast of distorted picture2 (NR)

Figure 5-1 (a) of the original image 1, (b) to damage the image 1, (c) the model for the TV image restoration effect, (d) model for image restoration Criminisi effect. Figure 5-2 (a) of the damaged image 2, (b) the model for the TV image restoration effect, (c) the effect of Criminisi model image restoration. Figure 5-2 experiments picture does not exist the original image. 1 is a small area where the image restoration, image 2 is a large area of repair.

Table 5-1 for repairing damaged image comparison algorithm and its quality evaluation algorithms 1 and 2. Where MSE and PSNR for full reference quality assessment, NR-NIQE without reference quality assessment, subjective quality 
assessment results to repair the human eye observation.

Table1 5-1 Repair and evaluation algorithm compares

\begin{tabular}{|c|c|c|c|c|c|c|}
\hline $\begin{array}{c}\text { Evaluation } \\
\text { algorithm }\end{array}$ & \multicolumn{2}{|c|}{$\begin{array}{c}\text { Evaluation of } \\
\text { restoration algorithm } \\
\text { of TV }\end{array}$} & $\begin{array}{c}\text { 20 } \\
\text { restoration algorithm } \\
\text { of Criminisi }\end{array}$ & $\begin{array}{c}\text { Relation } \\
\text { between } \\
\text { score and } \\
\text { image } \\
\text { quality }\end{array}$ & NF \\
\cline { 2 - 6 } & image1 & image2 & image1 & image2 & Negative & no \\
\hline MSE & 236.9344 & & 41.4362 & & Positive & no \\
\hline PSNR & 24.3845 & & 31.9570 & & Negative & yes \\
\hline NR-NIQE & 5.5087 & 4.2455 & 4.6892 & 2.6797 & yes \\
\hline $\begin{array}{c}\text { subjective } \\
\text { quality } \\
\text { assessment }\end{array}$ & low & low & high & high & & \\
\hline $\begin{array}{c}\text { Consume } \\
\text { time }\end{array}$ & $6.6324 \mathrm{~s}$ & $\begin{array}{c}6.7534 \\
\mathrm{~s}\end{array}$ & $\begin{array}{c}38.5885 \\
\mathrm{~s}\end{array}$ & $\begin{array}{c}27.7569 \\
\mathrm{~s}\end{array}$ & & \\
\hline
\end{tabular}

By comparing the two algorithms can be found in the chart, TV repair methods more used to repair damaged small-scale image restoration relatively fast; but for large-scale, rich texture damaged image, its effect is not ideal repair, easy to produce blurred effect. The repair effect Criminisi algorithm is relatively TV algorithm is more excellent, rendering almost imperceptible traces of repair, very close to the original, but this algorithm for minimal noise and restorative effects linear repair area is not good, and this is its principle block units for matching patch of the decision, and very time consuming. And the evaluation of image quality can basically meet the subjective quality evaluation criteria.

\section{Conclusion}

For image restoration algorithms, this paper simulation-based variational PDE structural repair and patching algorithm based on texture synthesis block Criminisi repair algorithm, combined with subjective and objective evaluation of image quality evaluation criteria to quantify the quality of image restoration, in order to further improve the image restoration precision and accuracy. But this article repair algorithm and evaluation algorithm also need to further improve fitness and systematic. With the continuous development of digital image technology, people are increasingly demanding high image quality, more efficient image restoration technology will follow the progress, widely used in the field of image restoration.

Fund: Peng Xiaobo, the Shenzhen Municipal Science and Technology R \& $\mathrm{D}$ funding technology research project (project number: JCYJ20120613162656264); Feng Ping, Shenzhen Science and Technology Innovation Council (item number: JCYJ20140418091413575)

\section{References:}

[1] ZHANG Hong-ying. Research and Application of digital image restoration 
technology [D]. Chengdu: University of Electronic Science and Technology, 2006.

[2] Shao Xiaowei, Liu Zheng Kai, Song Choi. An adaptive image restoration method TV model. Circuits and Systems, 2004, 9 (2)

[3] Li Junbo, THE ACADEMY, wearing yellow animal husbandry, good philosophy. Improved Criminisi image restoration algorithms. "Industrial Control Computer" 2014.

[4] Dai Shimei, ZHANG Hong-ying, who over. A fast image restoration algorithm based on a sample of [J]. ITS applications, 2010,29 (22).

[5]A. Mittal, A. K. Moorthy, and A. C. Bovik, "No-reference image quality assessment in the spatial domain,” IEEE Trans. Image Process.,2012, to be published.

[6] Anish Mittal, Rajiv Soundararajan, and Alan C. Bovik, Fellow, IEEE. Making a "Completely Blind" Image Quality Analyzer. SIGNAL PROCESSING LETTERS, VOL. 20, NO. 3, MARCH 2013.

[7] Linhai Xiang, Zhang Xin. No reference image quality assessment reviewed. Computer Knowledge and Technology Computer Knowledge and Technology Vol.5, No.28, October 2009.

[8] Jianggang Yi, Huang Dajiang, Wang Xu. Advances in image quality evaluation methods [J]. Electronics \& Information Technology, 2010,32 (1).

[9] House Bin Shen Haibin, Zhao Wufeng, and so on. Evaluation based on natural image statistics without reference image quality [J]. Zhejiang University: Engineering Science, 2010,4 (2). 